\title{
PREDICTION ANALYSIS OF PHARMACOKINETIC PARAMETERS OF SEVERAL ORAL SYSTEMIC DRUGS USING IN SILICO METHOD
}

\author{
RADITYA ISWANDANA ${ }^{1}$, PERMATA AISYAH ${ }^{2}$, REZI RIADHI SYAHDI ${ }^{2 *}$ \\ ${ }^{1}$ Department of Pharmaceutics and Pharmaceutical Technology, Laboratory of Pharmaceutical Technology and Formulation, Faculty \\ of Pharmacy, Universitas Indonesia, Depok, 16424, Indonesia. ${ }^{2}$ Department of Biomedical Computation, Laboratory of Biomedical \\ Computation, Faculty of Pharmacy, Universitas Indonesia, Depok, 16424, Indonesia. Email: rezi.riadhi@farmasi.ui.ac.id
}

Received: 02 October 2019, Revised and Accepted: 24 December 2019

ABSTRACT

Objective: This research aims to observe the pharmacokinetic parameters that can be predicted using a software, discover the best software to predict pharmacokinetic properties, and analyze the correlation between pharmacokinetic parameters used as descriptors with absorption percentage (\%ABS) from references.

Methods: This research was conducted using Molinspiration, QikProp, admetSAR, SwissADME, Chemicalize, and pkCSM software. This research analyzed 34 oral systemic drug compounds for absorption rate and six descriptors comprising molecular weight (MW), logP, hydrogen bond acceptor (HBA), hydrogen bond donor (HBD), polar surface area (PSA), and pKa.

Results: SwissADME showed the most accurate prediction of MW, logP, and HBD. Chemicalize showed the most accurate prediction of HBA, PSA, and pKa. Further, admetSAR showed the most accurate prediction of Caco-2 permeability. The highest $\mathrm{R}$ value was obtained from the correlation between \%ABS with Caco-2 permeability on 34 drug compounds $(\mathrm{R}=0.8211)$.

Conclusion: The highest $\mathrm{R}$ value was obtained from the correlation between \%ABS with Caco2 permeability on 34 drug compounds (R=0.8211), which showed a significant relationship $\left({ }^{*} \mathrm{p}<0.001\right)$. This indicates that oral systemic drugs are affected by Caco- 2 permeability. Moreover, the result of this research can be considered for the development of oral systemic drugs.

Keywords: Absorption percentage, Absorption, distribution, metabolism, and excretion prediction, In silico, Oral systemic drugs, Physicochemical parameters, Pharmacokinetic parameters.

(C) 2020 The Authors. Published by Innovare Academic Sciences Pvt Ltd. This is an open access article under the CC BY license (http://creativecommons. org/licenses/by/4. 0/) DOI: http://dx.doi.org/10.22159/ijap.2020.v12s1.FF057

\section{INTRODUCTION}

Oral administration is the most commonly used route for drug administration due to its convenience, high level of patient safety, and the relatively low production cost. For efficiency, drugs designed to be systemically active must be absorbed from the site of administration [1]. The effectiveness of oral systemic drugs is affected by pharmacokinetic properties, involving absorption, distribution, metabolism, and excretion.

The major steps occurring during the absorption of oral drugs are the dissolution of the drug from the dosage form, the solubility of the drug, the drug's effective permeability to the intestinal mucosa, and the drug's pre-systemic metabolism [2]. Dissolution is the process by which a solid drug substance dissolves in a solvent over time [3]. Solubility is the mass of solute that dissolves in a specific mass or volume of solvent at a given temperature. Thus, the solubility test may be used to predict bioavailability.

Noyes-Whitney equation reveals that dissolution may be affected by the physicochemical characteristics of the drug, formulation, and solvent [4]. The permeation of drug across the gut wall (a model lipid membrane) is affected by the ability of the drug to diffuse $(D)$ and partition between the lipid membranes. Further, the aqueous solubility of the drug can be estimated by aqueous environments, depending on the ionization of the tested drug [5].

Furthermore, most drugs are weakly acidic or weakly basic compounds [6]. Weakly acidic and weakly basic compounds cannot completely ionize in aqueous media, which are appropriate because unionized drugs, as opposed to ionized drugs, tend to exhibit considerably greater lipid solubility. In addition to their effect on dissolution kinetics, the physicochemical properties of the drug such as pKa and $\mathrm{pH}$ profile, particle size, polymorphism, hygroscopicity, and partition coefficient are important properties in drug designing [3].

This study analyzed the absorption of drugs in the body using in silico method. The solubility and permeability of the intestine toward the drug are considered the two most important determinants of the bioavailability of oral drugs. Moreover, the bioavailability of the drug may be reduced by efflux mechanism or first-pass metabolism in the intestine and/or liver. This study aimed to observe the pharmacokinetic parameters that can be predicted using software, discover the best software to predict pharmacokinetic properties, and analyze the correlation between pharmacokinetic parameters used as descriptors with absorption percentage (\%ABS) from reference.

\section{METHODS}

Hardware and software

Two computers with the following specifications were used. The first computer had a Quad-Core Processor CPU Q9400 @ 2.67 GHz (Intel ${ }^{\circledR}$ Core TM, America), system type 64-bit operating system, and Windows operating system. The second computer had an Intel ${ }^{\circledR}$ Core $^{\mathrm{TM}}$ i5-4210U CPU @ 1.70GHz (4 CPUs), 2.4GHz, 8192MB RAM, and Windows 10 Home 64-bit (10.0, Build 16299).

The software used was admetSAR (Shanghai Key Laboratory of New Drug Design, China) [7], SwissADME (Swiss Institute of Bioinformatics, Swiss) [8], QikProp (Schrodinger, LLC, New York, United States of America)[9], Chemicalize (ChemAxon Ltd., Budapest, 
Hungary) [10], pkCSM (Biosig Lab, University of Melbourne, Australia) [11], Molinspiration (Molinspiration Cheminformatics, Slovak Republic) [12], and Microsoft Excel.

\section{Drug compounds}

The drug compounds used in this research were two-dimensional structures of aminopyrine, cimetidine, ciprofloxacin, cromolyn sodium, cyclosporin, dexamethasone, doxorubicin, famotidine, fenoterol, hydrocortisone, ibuprofen, indomethacin, isoxicam, ketorolac, lansoprazole, lornoxicam, meloxicam, metaproterenol, methotrexate, methylprednisolone, naproxen, nizatidine, omeprazole, oxatomide, piroxicam, prednisolone, ranitidine, salicylic acid, sulindac, sumatriptan, tenidap, tenoxicam, terbutaline, and theophylline. The two-dimensional structures of the 34 drug compounds were downloaded from PubChem.

\section{Preparation of two-dimensional drug compounds}

The preparation of the structures of the drug compounds includes searching, selecting, downloading, and converting the structures from two-dimensional to three-dimensional; further, these drug compound structures were prepared using the information obtained from the database and bioinformatics website PubChem and MarvinSketch.

\section{Preparation of experimental pharmacokinetic parameters}

The pharmacokinetic parameters that were evaluated were \%ABS, molecular weight (MW), $\log \mathrm{P}$, hydrogen bond acceptor (HBA), hydrogen bond donor (HBD), polar surface area (PSA), pKa, and Caco-2 permeability. The data were collected from Zhao et al. [13] and previous researches [14-33].

\section{Validation of predicted pharmacokinetic parameters}

Validation was performed to predict the pharmacokinetic parameters (MW, logP, HBA, HBD, PSA, pKa, and Caco-2 permeability) of the 34 drug compounds using Molinspiration, admetSAR, SwissADME, QikProp, Chemicalize, and pkCSM.

\section{Optimization of predicted pharmacokinetic parameters}

By comparing experimental data from the reference with the softwarepredicted data from multiple software, optimization was performed to determine the software that showed the most accurate prediction of the pharmacokinetic parameters used in this research.

\section{Analysis predicted descriptors for oral systemic drugs}

The experimental \%ABS was correlated with the predicted pharmacokinetic parameters and analyzed using Microsoft Excel. The resulting scatter plot showed the correlation coefficient (R) between the experimental \%ABS with the descriptors of oral systemic drugs. Furthermore, SPSS was used to calculate significant values $\left(^{*} p\right)$. If ${ }^{*}$ p-value was $<0.05$, the result was considered statistically significant.

\section{RESULTS AND DISCUSSION}

Preparation of experimental pharmacokinetic parameters

Experimental pharmacokinetic parameters were obtained from the study by Zhao et al. and previous studies (Table 1 ).

\section{Validation of predicted pharmacokinetic parameters}

MW, $\log$, HBA, HBD, and PSA can be predicted using QikProp, SwissADME, Molinspiration, and Chemicalize. Further, pKa can be predicted using Chemicalize. Caco2 permeability can be predicted using QikProp, admetSAR, and pkCSM.

Optimization of predicted pharmacokinetic parameters

SwissADME is the most accurate software in predicting MW, logP, and HBD. Chemicalize is the most accurate software in predicting HBA,

Table 1: Reference prediction pharmacokinetic parameters

\begin{tabular}{|c|c|c|c|c|c|c|c|c|c|}
\hline Drugs & $\% \operatorname{ABS}(\mathrm{g} / \mathrm{mol})^{\mathrm{a}}$ & $\mathbf{M W}^{\mathbf{b}}$ & $\log P^{c}$ & HBA $^{\text {d }}$ & HBD $^{\mathbf{e}}$ & LR5 $^{f}$ & PSA $\left(\AA^{2}\right)^{g}$ & $\mathbf{p K a}^{\mathrm{h}}$ & Caco2 permeability $\left(10^{-6} \mathrm{~cm} / \mathrm{s}\right)^{\mathrm{i}}$ \\
\hline Aminopyrine & 100 & 231 & 1 & 4 & 0 & $\checkmark$ & 25 & 5.00 & -4.44 \\
\hline Cromolyn sodium & 0.4 & 468 & 1.92 & 11 & 3 & $\checkmark$ & 167 & - & -6.89 \\
\hline Ibuprofen & 95 & 206 & 3.5 & 2 & 1 & $\checkmark$ & 40 & 4.60 & -4.58 \\
\hline Indomethacin & 100 & 358 & 4.27 & 5 & 1 & $\checkmark$ & 68 & 4.50 & -4.89 \\
\hline Isoxicam & 100 & 335 & 2.83 & 8 & 2 & $\checkmark$ & 116 & - & -5.61 \\
\hline Ketorolac & 90 & 225 & 1.62 & 4 & 1 & $\checkmark$ & 62 & 4.47 & - \\
\hline Lornoxicam & 100 & 372 & 3.15 & 7 & 2 & $\checkmark$ & 100 & 4.70 & - \\
\hline Naproxen & 99 & 230 & 3.34 & 3 & 1 & $\checkmark$ & 51 & 4.27 & -4.66 \\
\hline Oxatomide & 100 & 426 & 5.41 & 5 & 1 & $\checkmark$ & 44 & - & - \\
\hline Piroxicam & 100 & 331 & 1.98 & 7 & 2 & $\checkmark$ & 99 & 5.28 & -4.33 \\
\hline Salicylic acid & 100 & 138 & 2.26 & 3 & 2 & $\checkmark$ & 55 & 2.85 & -4.82 \\
\hline Sulindac & 90 & 356 & 2.81 & 3 & 1 & $\checkmark$ & 58 & 4.70 & - \\
\hline Tenidap & 89 & 321 & 0.63 & 5 & 3 & $\checkmark$ & 77 & 4.50 & -4.57 \\
\hline Tenoxicam & 100 & 337 & 2.42 & 7 & 2 & $\checkmark$ & 100 & 4.42 & - \\
\hline Theophylline & 100 & 180 & -0.02 & 6 & 1 & $\checkmark$ & 64 & 8.51 & -4.61 \\
\hline Doxorubicin & 12 & 543 & 0.1 & 12 & 7 & alert & 204 & 8.20 & -6.48 \\
\hline Cyclosporine & 28 & 1202 & 3.8 & 23 & 5 & alert & 324 & - & -6.05 \\
\hline Methotrexate & 70 & 454 & -0.3 & 13 & 7 & alert & 211 & 3.30 & -6.1 \\
\hline Lansoprazole & 85 & 369 & 3.07 & 5 & 1 & $\checkmark$ & 65 & 3.83 & - \\
\hline Omeprazole & 80 & 345 & 2.23 & 6 & 1 & $\checkmark$ & 72 & 4.25 & - \\
\hline Dexamethasone & 80 & 392 & 2.01 & 5 & 3 & $\checkmark$ & 90 & 6.00 & -4.91 \\
\hline Hydrocortisone & 91 & 362 & 1.61 & 5 & 3 & $\checkmark$ & 96 & - & -4.82 \\
\hline Methylprednisolone & 82 & 374 & 1.96 & 5 & 3 & $\checkmark$ & 95 & - & -4.93 \\
\hline Prednisolone & 99 & 360 & 1.62 & 5 & 3 & $\checkmark$ & 97 & - & -4.72 \\
\hline Cimetidine & 64 & 252 & 0.4 & 6 & 3 & $\checkmark$ & 84 & 6.93 & -5.9 \\
\hline Famotidine & 38 & 337 & -0.57 & 9 & 8 & $\checkmark$ & 182 & 6.74 & -6.16 \\
\hline Nizatidine & 90 & 331 & 0.5 & 7 & 2 & $\checkmark$ & 83 & 13.23 & - \\
\hline Ranitidine & 64 & 314 & 0.27 & 7 & 2 & $\checkmark$ & 82 & 13.26 & -6.31 \\
\hline Metaproterenol & 44 & 211 & 0.08 & 4 & 4 & $\checkmark$ & 81 & 4.30 & -6.42 \\
\hline Terbutaline & 62 & 225 & 0.08 & 4 & 4 & $\checkmark$ & 80 & 8.79 & -6.16 \\
\hline
\end{tabular}

${ }^{\mathrm{a} A b s o r p t i o n}$ data were taken from Reference [13], ${ }^{\mathrm{b}}$ molecular weight data were taken from reference [13], , logP data were taken from Reference [13], ${ }^{\mathrm{d}}$ hydrogen bond acceptor (HBA) data were taken from reference [13], ehydrogen bond donor (HBD) data were taken from reference [13], fLipinski's rule of five (RO5) data were taken from Reference [13]. Checkmark $(\checkmark)$ means the compound fulfilled the rule, spolar surface area (PSA) data were taken from reference [13], hpKa data were taken from reference [14-33], 'Caco2 permeability data were taken from reference [34,35], 'No data, ABS: Absorption 
PSA, and pKa. Furthermore, admetSAR is the most accurate software in predicting Caco 2 permeability. Fig. 1 shows the correlation between experimental data and predicted data.

The correlation between reference MW and predicted MW showed $\mathrm{R}=0.9985$; the correlation between reference $\log \mathrm{P}$ and predicted $\log \mathrm{P}$ showed $\mathrm{R}=0.8694$; the correlation between reference HBA and predicted HBA showed $\mathrm{R}=0.8716$; the correlation between reference HBD and predicted HBD showed $\mathrm{R}=0.9253$; the correlation between reference PSA and predicted PSA showed $\mathrm{R}=0.9916$; the correlation between reference $\mathrm{pKa}$ and predicted $\mathrm{pKa}$ showed $\mathrm{R}=0.6463$; and the correlation between reference Caco- 2 permeability and predicted Caco2 permeability showed $\mathrm{R}=0.8593$.

\section{Analysis predicted descriptors for oral systemic drugs}

The correlation between \%ABS and predicted pharmacokinetic parameters was analyzed using Microsoft Excel. The correlation between \%ABS and predicted MW showed $\mathrm{R}=-0.4773$; the correlation $\% \mathrm{ABS}$ percentage and predicted $\log \mathrm{P}$ showed $\mathrm{R}=0.3534$; the correlation between \%ABS and predicted HBA showed $\mathrm{R}=-0.7205$; the correlation between \%ABS and predicted HBD showed $\mathrm{R}=-0.7046$; the correlation between $\% \mathrm{ABS}$ and predicted PSA showed $\mathrm{R}=-0.6627$; the correlation between \%ABS and predicted $\mathrm{pKa}$ showed $\mathrm{R}=-0.5453$; and the correlation between \%ABS and predicted Caco-2 permeability showed $\mathrm{R}=0.8211$ (Fig. 2).

Table 2 indicates the two absorption multiple regression models obtained in this research. Model 1 was created with all compounds with complete parameters and model 2 was created with all compounds with complete parameters but without $100 \%$ absorption. From the data, multiple regressions derived better $R^{2}$ value were obtained from model 2 than from model 1 (0.792948 and 0.750249, respectively). However, because the standard errors for the models were similar (17.22067 and 17.57382, respectively), the differences were not statistically significant. Further, the weightages of several parameters in model 2 were larger than those in model 1 and $\log$, Caco2, and pKa were noticeably larger than the others. Absorption multiple regression results are listed in Table 2.

\section{DISCUSSION}

The correlation between reference MW and predicted MW; reference $\log \mathrm{P}$ and predicted $\log \mathrm{P}$; reference HBA and predicted HBA; reference HBD and predicted HBD; reference PSA and predicted PSA; and reference Caco-2 permeability and predicted Caco-2 permeability showed strong correlations with $\mathrm{R}=0.9985,0.8694,0.8716,0.9253$, 0.9916 , and 0.8593 , respectively; however, the correlation between reference $\mathrm{pKa}$ and predicted $\mathrm{pKa}$ showed medium correlation with $\mathrm{R}=0.6463$. Therefore, predicted pKa showing $\mathrm{R}$ value $(<0.7)$ is the only parameter that exhibits a moderate positive relationship [36]. Several researches mention the accuracy problem of pKa prediction and state that pKa prediction is highly dependent on the dataset [37]. The simplification of the software calculation may also be a limitation of pKa prediction [38]. To improve the algorithm, drug type clustering based on its pKa level should be considered because the algorithm may show different results for acidic and basic drugs. The pKa range of clusterization should be optimized in further research. In addition, the dataset in this experiment contains various compounds that may act as obstacles in accurate pKa prediction for all structures. In this study, pKa of several compounds, such as aminopyrine with anti-inflammatory action; hydrocortisone, methylprednisolone, and prednisolone, which are corticosteroid agents; and nizatidine and ranitidine from $\mathrm{H} 2$ receptor antagonist group, could not be accurately predicted. The $\mathrm{R}$ value is suggested to reach $>0.9$ to be considered as accurate prediction.

From this research, we found that the various software programs provided different parameter prediction results. None of the software served as the most accurate prediction tool for all parameters. However, out of seven parameters, Chemicalize and SwissADME accurately predicted three complimentary parameters each. Moreover, Caco2 prediction only can be accurately done using admetSAR.
Analysis descriptors for the 34 oral systemic drugs resulting in the highest $\mathrm{R}$ value were the significant correlation between \%ABS and Caco-2 permeability $\left(\mathrm{R}=0.8211 ;{ }^{*} \mathrm{p}<0.001\right)$ (Fig. 2$)$.

The absorption multiple regression models were derived from these data by including the compounds with $100 \%$ absorption (model 1 ) or excluding it (model 2) to observe how the nonlinear function part affects the correlation. Better $\mathrm{R}^{2}$ values were obtained from model 2 than from model 1; however, the difference was not significant. Further, the weightages of several parameters in model 2 were larger than those in model 1, with LogP, Caco2, and pKa being noticeably larger than the others. This suggests that these three parameters, as opposed to MW and PSA, have higher tendencies to affect absorption.

In general, a model is acceptable if it has $\mathrm{R}^{2}>0.6$ [39]. In addition, in this case, good model fitness was observed in both models. This study is limited by its small dataset and usually good prediction is statistically derived from large datasets; therefore, further considerations need to be undertaken such as to selectively include various drugs and also to try several other software programs not included in this study. Nevertheless, from the experiment, both models are acceptable to be used as early in silico tools to assist the prediction of the absorption of systemic oral drugs.

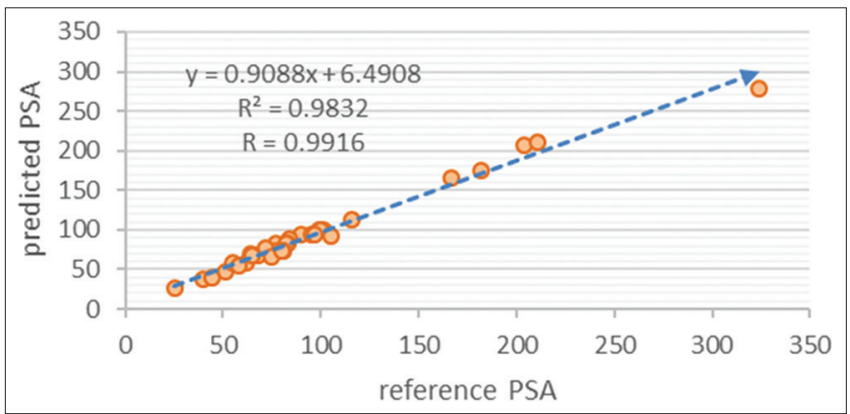

Fig. 1: Scatter plot reference polar surface area (PSA) [4] versus predicted PSA

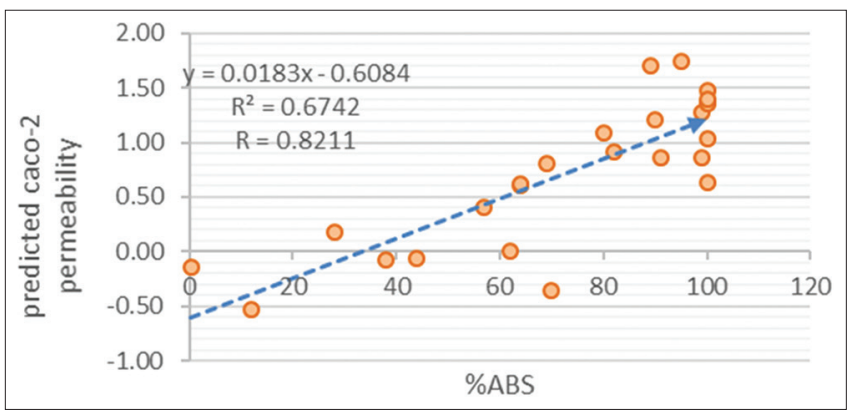

Fig. 2: Scatter plot absorption percentage versus predicted Caco-2 permeability

Table 2: Multiple regression model of absorption made from calculated parameters

\begin{tabular}{lllll}
\hline No. & Model & $\mathbf{R}^{2}$ & $\mathbf{n}$ & SE \\
\hline 1 & \%ABS=0.002556 MW-1.61419 & 0.750249 & 26 & 17.22067 \\
& LogP-7.67912 HBA-3.92035 & & & \\
& HBD+0.187031 PSA-1.38116 & & & \\
& pKa+13.23259 Caco2+108.3029 & & & \\
2 & \%ABS=0.00649 MW-5.56972 & 0.792948 & 18 & 17.57382 \\
& LogP-8.45619 HBA+2.141478 & & & \\
& HBD+0.205398 PSA-2.33053 & & & \\
& pKa+25.98339 Caco2+93.12721 & & & \\
\hline
\end{tabular}

MW: Molecular weight, HBA: Hydrogen bond acceptor, HBD: Hydrogen bond donor, PSA: Polar surface area, ABS: Absorption 


\section{CONCLUSION}

Parameter prediction was successfully performed in this research. SwissADME was the most accurate software in predicting MW, $\log$, and HBD; Chemicalize was the most accurate software in predicting HBA, PSA, and pKa; and admetSAR was the most accurate software in predicting Caco2 permeability. The highest $\mathrm{R}$ value was obtained from the significant correlation between \%ABS and Caco-2 permeability of 34 drug compounds $\left(\mathrm{R}=0.8211 ;{ }^{*} \mathrm{p}<0.001\right)$. These results indicate that the $\% \mathrm{ABS}$ of oral systemic drugs is affected by Caco-2 permeability.

\section{ACKNOWLEDGMENT}

The authors would like to thank Direktorat Riset dan Pengabdian Masyarakat Universitas Indonesia for Hibah PITTA UI for this research.

\section{CONFLICTS OF INTEREST}

All authors have none to declare.

\section{REFERENCES}

1. Engman H. Intestinal barriers to oral drug absorption. Analysis 2003;9:1-62.

2. Song NN, Zhang S, Liu C. Overview of factors affecting oral drug absorption. Asian J Drug Metab Pharmacokinet 2004;4:167-76

3. Shargel L, Wu-Pong S, Yu A. Applied Biopharmaceutics and Pharmacokinetics. Annals of Internal Medicine. $5^{\text {th }}$ ed., Vol. 94. New York: McGraw Hill; 2004. p. 826.

4. Beringer P. Remington: The Science and Practice of Pharmacy. Philadelphia, PA: Lippincott Williams and Wilkins; 2011. p. 675.

5. Shekhawat PB, Pokharkar VB. Understanding peroral absorption: Regulatory aspects and contemporary approaches to tackling solubility and permeability hurdles. Acta Pharm Sin B 2017;7:260-80.

6. Manallack DT. The $\mathrm{pK}$ (a) distribution of drugs: Application to drug discovery. Perspect Medicin Chem 2007;1:25-38.

7. Cheng F, Li W, Zhou Y, Shen J, Wu Z, Liu G, et al. AdmetSAR: A comprehensive source and free tool for assessment of chemical ADMET properties. J Chem Inf Model 2012;52:3099-105.

8. Daina A, Michielin O, Zoete V. SwissADME: A free web tool to evaluate pharmacokinetics, drug-likeness and medicinal chemistry friendliness of small molecules. Sci Rep 2017;7:42717.

9. QikProp Ver. 3.5. New York: Schrödinger LLC; 2012. p. 6. Available from: http://www.gohom.win/ManualHom/Schrodinger/ Schrodinger_2012_docs/qikprop/qikprop_user_manual.pdf

10. Swain M. Chemicalize.org. J Chem Inf Model 2012;52:613-5.

11. Pires DE, Blundell TL, Ascher DB. PkCSM: Predicting small-molecule predicting small-molecule pharmacokinetic and toxicity properties using graph-based signatures. J Med Chem 2015;58:4066-72.

12. Calculation of Molecular Properties and Bioactivity Score; 2018. Available from: http://www.molinspiration.com/cgi-bin/properties. [Last accessed on 2020 Mar 27].

13. Zhao YH, Abraham MH, Le J, Hersey A, Luscombe CN, Beck G, et al. Rate-limited steps of human oral absorption and QSAR studies. Pharm Res 2002;19:1446-57.

14. Lau ET, Giddings SJ, Mohammed SG, Dubois P, Johnson SK, Stanley RA, et al. Encapsulation of hydrocortisone and mesalazine in zein microparticles. Pharmaceutics 2013;5:277-93.

15. Takahashi K, Sakano H, Rytting JH, Numata N, Kuroda S, Mizuno N. Influence of $\mathrm{pH}$ on the permeability of p-toluidine and aminopyrine through shed snake skin as a model membrane. Drug Dev Ind Pharm 2001;27:159-64

16. Fang B, Li P, Shi X, Chen F, Wang L. Incompatibilities of lornoxicam with 4 antiemetic medications in polyole fi $\mathrm{n}$ bags during simulated intravenous administration. Medicine (Baltimore) 2016;95:1-5 .

17. Avdeef A, Berger CM. pH-metric solubility. 3. Dissolution titration template method for solubility determination. Eur J Pharm Sci 2001;14:281-91.

18. Rodríguez-Barrientos D, Rojas-Hernández A, Gutiérrez A, Moya-
Hernández R, Gómez-Balderas R, Ramírez-Silva MT. Determination of pKa values of tenoxicam from $1 \mathrm{H}$ NMR chemical shifts and of oxicams from electrophoretic mobilities (CZE) with the aid of programs SQUAD and HYPNMR. Talanta 2009;80:754-62.

19. Oliveira ÉD, Azevedo RD, Bonfilio R, De Oliveira DB, Ribeiro GP, De Araújo MB. Dissolution test optimization for meloxicam in the tablet pharmaceutical form. Braz J Pharm Sci 2009;45:67-73.

20. Juranic I, Dzeletovic D, Jovanovic J. Protolytic constants of nizatidine, ranitidine and $\mathrm{N}, \mathrm{N}$ '-DIMETHYL-2-nitro-1, 1-ethenediamine. Spectrophotometric and theoretical investigation. J Pharm Biomed Anal 2015;15:1-18.

21. Ahmadi F, Karamian E. Computational aided-molecular imprinted polymer design for solid phase extraction of metaproterenol from plasma and determination by voltammetry using modified carbon nanotube electrode. Iran J Pharm Res 2014;13:417-29.

22. Sheshala R. Validated high performance liquid chromatography (HPLC) method for the determination of sumatriptan in rabbit plasma: Application to pharmacokinetic study. Afr J Pharm Pharmacol 2012;6:98-107.

23. Nidhi $\mathrm{K}$, Indrajeet $\mathrm{S}$, Khushboo $\mathrm{M}$, Gauri $\mathrm{K}$, Sen DJ. Hydrotropy: A promising tool for solubility enhancement: A review. Int J Drug Dev Res 2011;3:26-33.

24. Gao H, Yao L, Mathieu HW, Zhang Y, Maurer TS, Troutman MD, et al. In silico modeling of nonspecific binding to human liver microsomes. Pharmacology 2008;36:2130-5.

25. Al-Deen AA, Dayo A, Ghoto MA, Arain MI, Parveen AQ. In vitro study of stability, quality and quantity of some clinically and non-clinically used cortisones from pharmaceutical preparations. Int J Biol Pharm Allied Sci 2014;3:2720-33.

26. Du-Cuny L. Aqueous Solubility of Drug-like Compounds (Doctoral Dissertation, Universitäts-und Landesbibliothek Bonn). Univ Darmstadt; 2006.

27. Ahmad T. Modeling of ibuprofen II: Effect of $\mathrm{pH}$ on the adsorption behavior on reversed phase liquid chromatography. Int J Appl Sci Technol 2012;2:49-56.

28. Fillet M, Bechet I, Piette V, Crommen J. Separation of nonsteroidal anti-inflammatory drugs by capillary electrophoresis using nonaqueous electrolytes. Electrophoresis 1999;20:1907-15.

29. Comer JE, Manallack D. Ionization constants and ionization profiles. Ref Modul Chem Mol Sci Chem Eng 2014; 8:357-97.

30. Raval G. Thermodynamic and Spectroscopic Studies on the Molecular Interaction of Doxorubicin (DOX) with Negatively Charged Polymeric Nanoparticles. University Toronto Master's Theses; 2012. p. 65.

31. Margalit E, Kugler LJ, Brumm MV, Meza JL, Kompella UB, Escobar ER, et al. The safety of intraocular ketorolac in rabbits. Investig Ophthalmol Vis Sci 2006;47:2093-9.

32. Roche VF. The chemically elegant proton pump inhibitors. Am J Pharm Educ 2006;70:101

33. Yamashita F, Wanchana S, Hashida M. Quantitative structure/property relationship analysis of Caco-2 permeability using a genetic algorithmbased partial least squares method. Kyoto Univ 2002;91:2230-9.

34. Castillo-Garit JA, Marrero-Ponce Y, Torrens F. Estimation of ADME properties in drug discovery: Predicting Caco-2 cell permeability using atom-based stochastic and non-stochastic linear indices. Int Electron Conf Synth Org Chem 2007;97:1-30.

35. Asuero AG, Sayago A, Gonz'alez AG. The correlation coefficient : An overview. The correlation coefficient : An overview. J Crit Rev Anal Chem 2006;36:41-59.

36. Settimo L, Bellman K, Knegtel RM. Comparison of the accuracy of experimental and predicted pKa values of basic and acidic compounds. Springer Sci 2013;31:86-8.

37. Lee AC, Crippen GM. Predicting pKa. Univ Michigan 2013;49:2013-33.

38. Frimayanti N, Yam ML, Lee HB, Othman R, Zain SM, Rahman NA. Validation of quantitative structure activity relationship (QSAR) model for photosensitizer activity prediction. Int J Mol Sci 2011;12:8626-44.

39. Alexander DL, Tropsha A, Winkler DA. Beware of R2: Simple, unambiguous assessment of the prediction accuracy of QSAR and QSPR models. J Chem Inf Model 2015;55:1316-22. 